\title{
A Classification Framework based on VPRS Boundary Region using Random Forest Classifier
}

\author{
Hemant Kumar Diwakar \\ Samrat Ashok Technological Institute, Vidisha
}

\author{
Sanjay Keer \\ Asst. Professor \\ Samrat Ashok Technological Institute, Vidisha
}

\begin{abstract}
Machine learning is a concerned with the design and development of algorithms. Machine learning is a programming approach to computers to achieve optimization .Classification is the prediction approach in data mining techniques. Decision tree algorithm is the most common classifier to build tree because of it is easier to implement and understand. Attribute selection is a concept by which be select more significant attributes in the given datasets. These proposed a novel hybrid approach combination of VPRS with Boundary Region and Random Forest algorithm called VPRS Boundary Region based Random Forest Classifier (VPRSBRRF Classifier) which is used to deal with uncertainties, vagueness and ambiguity associated with datasets. In this approach, select significant attributes based on variable precision rough set theory with boundary region as an input to Random Forest classifier for constructing the decision tree which is more efficient and scalable approach for classification of various datasets.
\end{abstract}

\section{Keywords}

Discretization, Variable Precision Rough Sets, Boundary Region, Random Forest

\section{INTRODUCTION}

A discretization algorithm is essential in order to handle problems with real-valued attributes with Decision Trees (DTs). The discretization of authentic value attributes is one of the consequential delicate situations to be solved in data mining, especially in rough set theory. Everyone knows that, when the value set to any attribute in a decision table is continuous value (real number), The discretization of authentic value attributes is one of the consequential quandaries or delicate situation to be solved in data mining, In such case, the number of equivalence classes based on that attribute will be enormous and there will be very less elements in each of such equivalence class, which will lead to the generation of a enormous number of rules in the classification of rough set, there for making rough set theoretic classifiers inefficient [2].Discretization is a process by which the grouping of values of the attributes in intervals in such a way that the knowledge content or the discernibility is not lost. There are many discretization approaches have been developed so far. Nguyen S. H had given some accurate and exhaustive detailed description about discretization in rough set in reference [1].

However, a very large proportion of real data sets include continuous variables: that is variables measured at the interval or ratio level. One solution to this dispute star is to partition numeric variables into a number of sub-ranges and treat each such sub-range as a type. This process of partitioning continuous variables into categories is usually termed discretization. Inappropriately, the number of ways to discretize a continuous attribute is infinite. Discretization is a potential time-consuming obstacle, since the number of possible discretizations is exponential in the number of interval threshold candidates within the area of expertise, [14].The aim of discretization is to find a set of cut points to partition the extent into a small number of intervals that have good class coherence, which is usually calculated by an evaluation function. Discretization is usually performed earlier to the learning process and it can be broken into two tasks. The $1^{\text {st }}$ job or chore is to find the number of discrete intervals. Only a with difficulty in any discretization algorithms perform this; often, the user must specify the number of intervals or provide a heuristic rule. The second chore is to find the width, or the boundaries, of the intervals given the range of values of a continuous attribute[2]

\subsection{Discretization Process}

The concept "cut-point" can be describe as mention to a real value within the range of continuous values that divides the range into two intervals, first interval is less than or equal to the cut point and the second interval is greater than the cutpoint. For instance, a continuous interval $[a, b]$ is divide into $[a, c]$ and $[c, b]$, where the value $\mathrm{c}$ is a cut-point value. Cutpoint value is also known as split-point. The explanation of a idea "arity" in the discretization context means the way of counting intervals or partitions. Earlier then discretization of a perpetual characteristic, arity can be set to $\mathrm{k}$ - the number of partitions in the perpetual characteristics. The highest, and utmost number of cut-points is $\mathrm{k}-1$. Discretization method reduces the arity but there is a trade-off between arity and its impact on the accuracy. A typical discretization method broadly consists of four steps: (1) sorting the perpetual values of the charactersticto be discretized, (2) evaluating a cut-point for splitting or adjacent intervals for bring or come together, (3) according to some criterion, splitting or bring or come together intervals of perpetual value, and (4) finally staying at some point. After sorting, the next step in the discretization method is to find the best "cut-point" to divides into parts a range of continuous values or the best pair of adjacent intervals to bring or come together. One typical evaluation function is to determine the correlation of a divide into a parts or a bring or come together with the class label. There are numerous evaluation functions found in the literature such as entropy calculate and statistical calculate (more details in the following sections). A stopping criterion specifies when to prevent the discretization process. It is usually ruled by a trade-off between lower arity with a better accepting but less accuracy and a higher arity with a poorer belief but higher accuracy. The count of inconsistencies (inconsistency is defined later) induce by discretization - it should not be much higher than the number of inconsistencies of the original data before discretization. Two conditions are considered or deliberate inconsistent if they are the same in their attribute values except for their class labels. Generally, the discretization approach can be placed into particular class or group or categorised as: (1) supervised or unsupervised, (2) 
global or local, (3) static or dynamic, (4) direct or incremental, (5) top down or bottom-up. These distinct categories in the following section.[13]

An approach first shipped by mathematician Zdzislaw Pawlak at the beginning of the eighties it is utilized as a mathematical implements to treat the nebulous and the imprecise. Rough Set Theory (RST) is similar to Fuzzy Set Theory, however the uncertain and imprecision in this approach is signify by a boundary region of a set, and not by a partial membership as in Fuzzy Set Theory. Rough Set conception can be defined generally by betokens of interior and closure topological operations ken approximations (Pawlak, 1982)

\subsection{Basic Notations of Rough Set}

In current years, rough set theory (RST), proposed by Pawlak [3], has attracted worldwide concentration of many researchers and expert.

\section{Definition 1.2.1: Information system.}

An information system is a quadruple IST $=(\mathrm{U}, \mathrm{A}, \mathrm{V}, \mathrm{F})$, where [10,11]:

(1) $U$ is a non-empty and finite set of objects;

(2) A is a non-empty and finite set of attributes;

(3) $\mathrm{V}$ is the union of attribute domains, i.e. $V=\mathrm{U}_{a \in A} \mathrm{~V}_{\mathrm{a}}$, where $\mathrm{Va}$ indicates the domain of attribute $\mathrm{a}$;

\section{Definition 1.2.2.: Indiscernibility relation}

Given a decision table DT $=(\mathrm{U}, \mathrm{C}, \mathrm{D}, \mathrm{V}, \mathrm{F})$, for any subset of attributes the B-indiscernibility relation U/IND(P)is defined by $[10,11]$

With any $\mathrm{P}$ there is an associated evenness relation IND (p):

$$
\operatorname{IND}(\mathrm{I})=\left\{(\mathrm{I}, \mathrm{J}) \in \mathrm{U}^{2} \mid \forall a \in \mathrm{I}, a(x)=a(J)\right.
$$

notice that this corresponds to the equal balancing relation ,to object will equal only if they have same value of their attributes in P.The partition of U explain by U/IND (P) which is simply the set of equal classes generated by IND $(\mathrm{P})$ :

$$
\mathrm{U} / \mathrm{IND}(\mathrm{I})=\otimes\{U / I N D(\{a\}) \mid a \in \mathrm{I}\}
$$

\section{Definition 1.2.3: Lower and Upper Approximations}

Suppose that $\mathrm{X} \subseteq \mathrm{U}, \mathrm{X}$ can be approx using only the information make suitable within $\mathrm{P}$ by constitute the $\mathrm{P}$-lower and P-upper approximations of the classical crisp set $\mathrm{X}$ :[5]

$$
\begin{aligned}
& \underline{R X}=\{\mathrm{I} \|[\mathrm{I}] \mathrm{p} \subseteq \mathrm{X}\} \\
& \bar{R} \mathrm{X}=\{\mathrm{I} \|[\mathrm{I}] \mathrm{p} \cap \mathrm{X} \neq \phi
\end{aligned}
$$

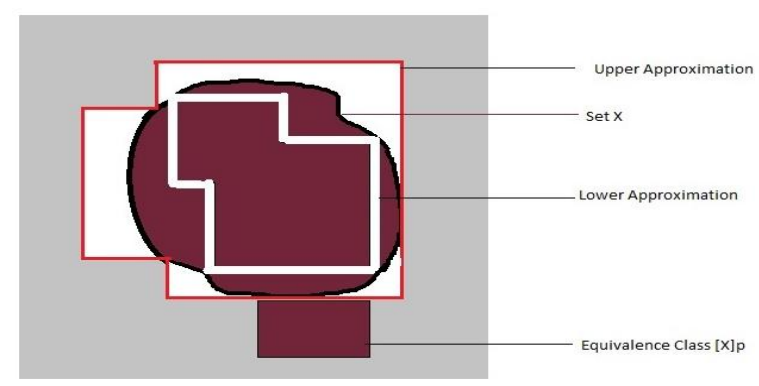

Figure 1
It is such a grouping of rows and columns $\{\underline{R} X, \bar{R} X\}$ that is termed an rough set. Study the approximation of abstraction $X$ in figure 1. Each square in the diagram represents an smoothness class; induce by indiscernibility enclosed by object values. utilizing the features in set $\mathrm{B}$, via these smoothness classes, the lower and upper approximations of $\mathrm{X}$ can be created. smoothness classes contained inside $\mathrm{X}$ exist to the lower approximation. Objects avoid an issue within this region can be say to exist definitely to concept $X$. smoothness classes within $\mathrm{X}$ and onward its boundary form the upper approximation. Those objects in this region can only be saying to possibly exist to the concept.

\section{Definition 1.2.4. Positive, Negative and Boundary Regions}

Let $\mathrm{P}$ and $\mathrm{I}$ be similarity relations over $\mathrm{U}$, then the positive [lower approximation], negative[upper approximation] and boundary regions are define as: [5]

$$
\begin{aligned}
& \operatorname{POSp}(\mathrm{I})=\bigcup_{i \in U / I} \underline{\mathrm{RX}} \\
& \operatorname{NEGp}(\mathrm{I})=\mathrm{U}-\bigcup_{i \in \overline{\mathrm{U}}} \overline{\mathrm{R}} \mathrm{X} \\
& \operatorname{BNDp}(\mathrm{I})=\bigcup_{\mathrm{i} \in \mathrm{U} / \mathrm{I}} \overline{\mathrm{R} X}-\bigcup_{i \in \mathrm{U} / \mathrm{I}} \underline{\mathrm{RX}}
\end{aligned}
$$

\section{Definition 1.2.5 Feature Dependency and Significance}

A critical in data analysis is examine dependencies place within by attributes. Apparently, a set of attributes I depends fully on a set of attributes $\mathrm{P}$, express by $\mathrm{P} \Rightarrow \mathrm{I}$, if every attribute values from I are differently or separately determine by values of attributes from $P$. If there, existent a functional dependency between or in middle of values I and P. Then I depend fully on P. In rough set theory, dependency is explain in the following way:

For $\mathrm{P}, \mathrm{I} \subset \mathrm{A}$, said that $\mathrm{I}$ depends on $\mathrm{P}$ in a degree $\mathrm{q}(0 \leq \mathrm{q} \leq \mathrm{l})$, denoted $\mathrm{P} \Rightarrow \mathrm{q}$ I, if

$q=\gamma_{\mathrm{p}}(I)=\frac{|\operatorname{POSp}(I)|}{|U|}$

where $\mathrm{jSj}$ position for the cardinality of set S.If $\mathrm{q}=1$, I depends fully on I, if $0<\mathrm{q}<1$, I depends partially (in a degree $\mathrm{q}$ ) on $\mathrm{P}$, and if $\mathrm{q}=0$ then I does not depend on $\mathrm{P}$ :

$$
\begin{aligned}
& \gamma_{\{\mathrm{b}, \mathrm{c}\}}(\{\mathrm{e}\})=\frac{\left|\operatorname{POS}_{\{b, c\}}(\{e\})\right|}{|U|} \\
& \sigma \mathrm{p}(\mathrm{I}, \mathrm{a})=\gamma_{\mathrm{p}}(\mathrm{I})-\gamma_{\mathrm{p}}(\mathrm{I})-\{\mathrm{a}\}^{(\mathrm{I})}
\end{aligned}
$$

\section{Definition 1.2.5 :Reducts}

For many application difficulties, it is often basic to maintain a compact form of the information system. One process to implement this is to search for a minimal representation of the original dataset. For this, the conception of a reduct is way out and defined as a minimal subset $\mathrm{R}$ of the initiatory feature set C such that for a given set of features $D, \gamma_{R}(D)=\gamma_{C}(D)$. [5]

\subsection{Variable Precision Rough Sets}

Variable precision rough sets (VPRS) [6] attempts to improve upon rough set theory by relaxing the subset operator. It was 
projected to analyze and identify data patterns which represent statistical rather than functional. The main idea of VPRS is to allow to be classified with an smaller than a certain predefined stratum. This approach is arguably relaxed to be understood within the framework of categorization Let X Y $\mathrm{U}$, the relative classification error is defined by[5].This approach apply on hiring data set given in table 1 .

\section{Table 1.Hiring Data Set}

\begin{tabular}{|c|c|c|c|c|c|}
\hline $\mathbf{U}$ & $\begin{array}{c}\text { Degre } \\
\mathbf{e}\end{array}$ & $\begin{array}{c}\text { Experienc } \\
\mathbf{e}\end{array}$ & $\begin{array}{c}\text { Englis } \\
\mathbf{h}\end{array}$ & $\begin{array}{c}\text { Referenc } \\
\mathbf{e}\end{array}$ & $\begin{array}{c}\text { Decisio } \\
\mathbf{n}\end{array}$ \\
\hline $\mathbf{1}$ & $\begin{array}{c}\mathrm{M} \\
\text { Tech }\end{array}$ & Medium & Yes & Excellent & Select \\
\hline $\mathbf{2}$ & $\begin{array}{c}\mathrm{M} \\
\text { Tech }\end{array}$ & Low & Yes & Neutral & Reject \\
\hline $\mathbf{3}$ & B.S.C & Low & Yes & Good & Reject \\
\hline $\mathbf{4}$ & M.S.C & High & Yes & Neutral & Select \\
\hline $\mathbf{5}$ & M.S.C & Medium & Yes & Neutral & Reject \\
\hline $\mathbf{6}$ & M.S.C & Medium & Yes & Excellent & Select \\
\hline $\mathbf{7}$ & $\begin{array}{c}\text { M } \\
\text { Tech }\end{array}$ & High & No & Good & Select \\
\hline $\mathbf{8}$ & B.S.C & Low & No & Excellent & Reject \\
\hline
\end{tabular}

This approach is easy to be understood within the framework of classification. Suppose $i, j \subseteq U$, the relative classification error is explain by:

$$
\mathrm{c}(\mathrm{I}, \mathrm{J})=1-\frac{|i \cap j|}{|i|}
$$

Observe that $\{\mathrm{c}(\mathrm{I}, \mathrm{J})=0\}$ only if $(\mathrm{I} \subseteq \mathrm{J})$. A degree of inclusion can be achieved by allowing a certain level of error, $\beta$ in classification:

$$
\mathrm{I} \subseteq{ }_{\beta} \mathrm{J} \text { iff } \mathrm{c}(\mathrm{I}, \mathrm{J}) \leq \beta, \quad 0 \leq \beta<0: 5
$$

Using $\subseteq{ }_{\beta}$ instead of $\subseteq$, the $\beta$-upper and $\beta$-lower approximations of a set $\mathrm{X}$ can be explain as:

$$
\begin{gathered}
\underline{R}_{\beta} I=\cup\left\{[\mathrm{x}]_{\mathrm{Q}} \in U / X \mid[x]_{\mathrm{Q}} \subseteq{ }_{\beta} I\right\} \\
\bar{R}_{\beta} \mathrm{I}=\mathrm{U}\left\{[x]_{\mathrm{Q}} \in \frac{U}{X} \mid c([x] \mathrm{Q}, I)<1-\beta\right\}
\end{gathered}
$$

Now notice, $\left(R_{\beta} I=\underline{R} \mathrm{I}\right)$ for $\beta=0$. the positive region ,negative region and boundary region are extended from original rough set theory.

$$
\begin{aligned}
& P O S_{Q, \beta}(\mathrm{I})=\underline{R}_{\beta} I \\
& N E G_{Q, \beta}(\mathrm{I})=\mathrm{U}-\bar{R}_{\beta} I \\
& B N D R_{Q, \beta}(I)=\underline{R}_{\beta} I-R_{\beta} I
\end{aligned}
$$

Table 2. Discretize Hiring Data Set

\begin{tabular}{|c|c|c|c|c|c|}
\hline $\mathbf{U}$ & $\begin{array}{c}\text { Degree } \\
(\mathbf{a})\end{array}$ & $\begin{array}{c}\text { Experience } \\
(\mathbf{b})\end{array}$ & $\begin{array}{c}\text { English } \\
(\mathbf{c})\end{array}$ & $\begin{array}{c}\text { Reference } \\
(\mathbf{d})\end{array}$ & Decision \\
\hline $\mathbf{1}$ & 0 & 1 & 1 & 2 & 1 \\
\hline $\mathbf{2}$ & 0 & 0 & 1 & 0 & 0 \\
\hline $\mathbf{3}$ & 1 & 0 & 1 & 1 & 0 \\
\hline $\mathbf{4}$ & 2 & 2 & 1 & 0 & 1 \\
\hline $\mathbf{5}$ & 2 & 1 & 1 & 0 & 0 \\
\hline
\end{tabular}

\begin{tabular}{|l|l|l|l|l|l|}
\hline $\mathbf{6}$ & 2 & 1 & 1 & 2 & 1 \\
\hline $\mathbf{7}$ & 0 & 2 & 0 & 1 & 1 \\
\hline $\mathbf{8}$ & 1 & 0 & 0 & 2 & 0 \\
\hline
\end{tabular}

Coming back to the example dataset in Table 1,first be convert hiring data set into a Discretize form as shown in table 2.Now equation 15 can be used to calculate the $\beta$ Boundary region for $\mathrm{Q}=\{\mathrm{b}, \mathrm{c}\}, \mathrm{X}=\{\mathrm{E}\}$ and $\beta=0.4$. giving $\beta$ to this value means that given set is considered to be a subset of another if they share about half the number of elements. The partitions of the universe of objects for Q and I are:

$$
\begin{gathered}
\mathrm{IND} / \mathrm{b}=\{2,3,8\},\{1,5,6\},\{4,7\} \\
\mathrm{IND} / \mathrm{c}=\{7,8\},\{1,2,3,4,5,6\}
\end{gathered}
$$

For every set $A \in U / R$ and $B \in U / R$, the value of $c(A, B)$ must be less than $\beta$ if the equivalence class $\mathrm{A}$ is to be contained in the $\beta$-positive region. Considering $\mathrm{A}=\{2\}$ gives

$$
\begin{aligned}
& \mathrm{C}\left(\mathrm{E}_{1}, \mathrm{X}_{1}\right)=\mathrm{C}(\{1,5,6\},\{1,4,6,7\})=1-\frac{|2|}{|3|}=0.334<\beta \\
& \mathrm{C}\left(\mathrm{E}_{1}, \mathrm{X}_{2}\right)=\mathrm{C}(\{1,5,6\},\{2,3,5,8\})=1-\frac{|1|}{|3|}=0.664>\beta
\end{aligned}
$$

So object $1,5,6$ is included to the $\beta$-boundary region as it is a $\beta$-subset of $\{2,3,5,8\}$ (and is in fact a traditional subset of the equivalence class). Taking $\mathrm{E}=\{2,3\}$, now a more interesting case is encountered:

$$
\begin{aligned}
& \mathrm{C}\left(\mathrm{E}_{2}, \mathrm{X}_{1}\right)=\mathrm{C}(\{2,3\},\{1,4,6,7\})=1-\frac{|\emptyset|}{|2|}=1>\beta \\
& \mathrm{C}\left(\mathrm{E}_{2}, \mathrm{X}_{2}\right)=\mathrm{C}(\{2,3\},\{2,3,5,8\})=1-\frac{|2|}{|2|}=0<\beta
\end{aligned}
$$

Here the objects 2,3 are contained in the $\beta$-boundary region . Calculating the subsets in this way leads to the following $\beta$ boundary region:

$$
\begin{gathered}
\underline{\mathrm{R}}_{(\mathrm{b}, \mathrm{c}) \beta} \mathrm{X}=\{1,2,3,4,5,6,7,8\} \\
\bar{R}_{(b, c) \beta} \mathrm{X}=\{1,2,3,4,5,6,7,8\} \\
B N R_{\beta}=0
\end{gathered}
$$

Now due to the relaxation of the subset operator. suppose a decision table ( $\mathrm{U}, C_{a} \cup D_{a}$ ), where $C_{a}$ is the set of conditional attributes and $D_{a}$ the set of decision attributes.

Where $\mathrm{Q}$ is also an similarity relation on $\mathrm{U}$. This can then be used to compute dependencies and thus decide $\beta$-reducts. The dependency function becomes:

$$
\begin{gathered}
\gamma_{Q, \beta}(\mathrm{I})=\frac{\left|B N D_{Q, \beta}(\mathrm{I})\right|}{|\mathrm{U}|} \\
\gamma_{(b, c) \beta}(X)=\frac{|0|}{|8|}=0 \\
\gamma_{(a, b, c) \beta}(X)=\frac{|2|}{|8|}=\frac{1}{4}
\end{gathered}
$$

Significant of hiring data set will be:

$$
\begin{gathered}
\sigma_{P(\theta, a)=\gamma_{P}(X)-\gamma_{P-\{a\}}} \\
\sigma_{(a)=\gamma_{(a, b, c)}(X)-\gamma_{(b, c)}(X)=} \sigma_{(a)=\frac{1}{4}-0=0.2} \\
\sigma_{(b)=\gamma_{(a, b, c)}(X)-\gamma_{(a, c)}(X)=} \sigma_{(b)=\frac{1}{4}-\frac{1}{4}=0}
\end{gathered}
$$




$$
\sigma_{(c)=\gamma_{(a, b, c)}(X)-\gamma_{(a, b)}(X)=\sigma_{(c)}=\frac{1}{4}-\frac{1}{4}=0}
$$

Here be can see a Quick reduct algorithm outlined earlier can be suitable to combine the reduction method built upon VPRS theory. By appling a suitable $\beta$ value to the algorithm, the $\beta$ lower approximation, $\beta$-positive region, and $\beta$ - dependency can take a place of the traditional calculations. This will result in a more almost accurate and exact final reduct, which may be a better generalization when encountering unseen data. Additionally, setting $\beta$ to 0 forces such a method to behave exactly like standard rough set theory.The extended classification of reducts in the VPRS approach found in [26, $27,28]$. However, the variable precision approach requires the supplementary and extra parameter $\beta$ which has to be specified from the start.

\section{PROPOSED METHODOLOGY}

\subsection{The Proposed System}

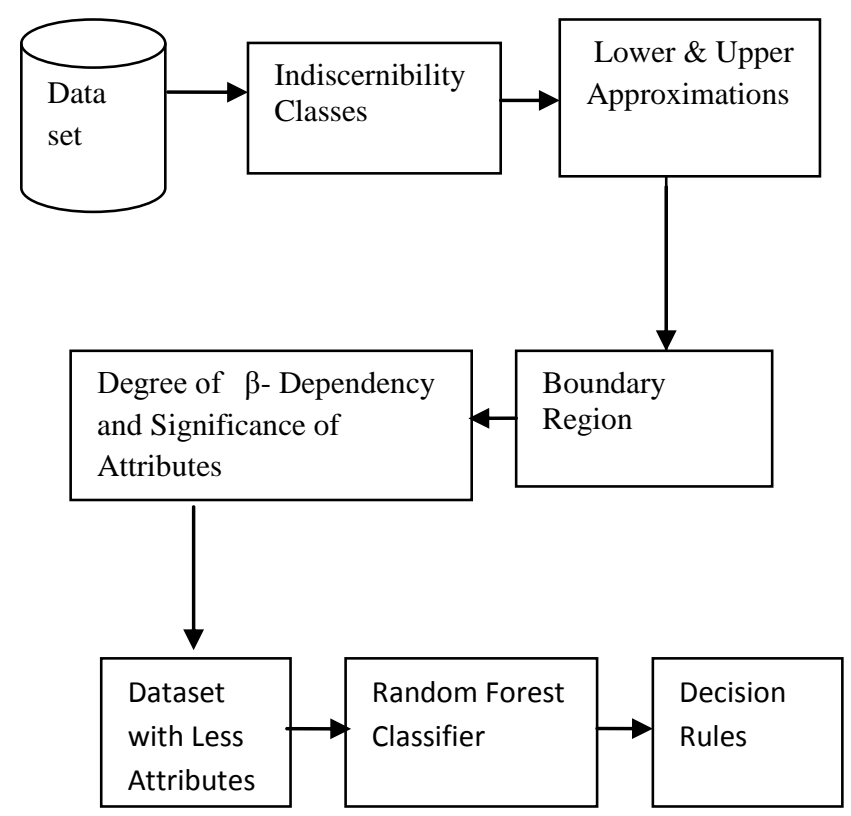

Figure 2: The Proposed System

\subsection{Consistency based Attribute Reduction Algorithm}

Input: An information system $I S=\left(U_{v} C \cup D_{v} V_{v} f\right)$

Output: One reduct $\mathrm{R}$ of the information system IS

Step 1: Compute the consistency $\delta_{C_{B} \beta}(D)$ based on VPRS boundary region.

Step 2: Let $R=\emptyset$

Step 3: Compute the core set $\operatorname{Core}(\mathrm{C})$ and $\mathrm{R}=\mathrm{R} \cup \operatorname{Core}(\mathrm{C})$

Step 4: To each attribute $a \in C-R$, compute $\operatorname{SGF}(a, R, D)$, let a1 represent the one that maximize $\operatorname{SGF}(\mathrm{a}, \mathrm{R}, \mathrm{D})$

Step 5: Add the selected attribute a1 to the subset $\mathrm{R}$, i.e. $\mathrm{R}=\mathrm{R}$ $\mathrm{U}$ a1

Step 6: If $\delta_{R_{\beta} \beta}(D)==\delta_{C \beta}(D)$, continue; otherwise goto step 4

Step 7: If $R \neq \operatorname{Cor} e(C)$, remove redundant attribute if exists Step 8: Output R.

\subsection{VPRSBRRF Classifier}

Now, propose our algorithm to generate a decision tree in the following way:

Input: An information system $I S=\left(U_{v} C \cup D_{v} V_{v} f\right)$

Output: A decision tree $T$.

Step 1: All labeled samples initially assigned to root node which is available in reduct $R$ of dataset

Step2: $\mathrm{N} \leftarrow$ root node

Step3: With node $\mathrm{N}$ do

$>$ Find the feature $\mathrm{F}$ among a random subset of features + threshold value T...

- ... that split the samples assigned to $\mathrm{N}$ into 2 subsets $S_{\text {left }}$ and $S_{\text {right } \cdots}$

-... so as to maximize the label purity within these subsets

$>\operatorname{Assign}(\mathrm{F}, \mathrm{T})$ to $\mathrm{N}$

$>$ If $\mathrm{S}_{\text {left }}$ and $\mathrm{S}_{\text {right }}$ too small to be splitted

- Attach child leaf nodes $\mathrm{L}_{\text {left }}$ and $\mathrm{L}_{\text {right }}$ to $\mathrm{N}$

- Tag the leaves with the most present label in $\mathrm{S}_{\text {left }}$ and $S_{\text {right }}$, respectively.

$>$ Else

- Attach child nodes $\mathrm{N}_{\text {left }}$ and $\mathrm{N}_{\text {right }}$ to $\mathrm{N}$

- Assign $S_{\text {left }}$ and $S_{\text {right }}$ to them, resp.

- Repeat procedure for $\mathrm{N}=\mathrm{N}_{\text {left }}$ and $\mathrm{N}=\mathrm{N}_{\text {right }}$

Step4: Random subset of features

- Random sketch repeated at each node

- For D-dimensional samples, usual subset size = round (sqrt (D)) (also round $(\log 2(\mathrm{x})))$

- $\rightarrow$ Increases variety among the rCARTs + reduces computational load

Step 5: Output the decision tree T.

\section{EXPERIMENTAL RESULTS AND ANALYSIS}

The implementation of the proposed VPRS with Boundary Region based Random Forest Classifier is provided. Therefore first the required tools and techniques are discussed then after the code implementation and development of the system is provided.

\subsection{The Datasets}

For analysing the results be have been used four data set from UGC . they are given below.

1. Iris Dataset[12]

2. Wine Data Set[12]

3. Breast Cancer Wisconsin (Original) Dataset[12]

4. Heart Disease [12]

\subsection{Accuracy}

Accuracy of proposed classification algorithm is a measurement of total accurate identified instances over the given samples. The accuracy of the classification can be 
evaluated on following datasets [12].

Table 3: Accuracy Comparisons between FID3 and VPRS Boundary Region based Random Forest Classifier

\begin{tabular}{|l|c|c|c|c|c|}
\hline Datasets & $\begin{array}{c}\text { Inst } \\
\text { anc } \\
\text { es }\end{array}$ & $\begin{array}{c}\text { Att } \\
\text { rib } \\
\text { utes }\end{array}$ & $\begin{array}{c}\text { FID3 } \\
\text { Accura } \\
\text { cy (\%) }\end{array}$ & $\mathbf{J 4 8}$ & $\begin{array}{c}\text { VPRSBRRF } \\
\text { Accuracy } \\
\text { (\%) }\end{array}$ \\
\hline Iris & 150 & 4 & $66.50 \%$ & $85 \%$ & $86 \%$ \\
\hline Wine & 178 & 14 & $74.70 \%$ & $79.2 \%$ & $91.01 \%$ \\
\hline $\begin{array}{l}\text { Breast } \\
\text { Cancer } \\
\text { Wisconsi } \\
\text { n } \\
\text { (Original } \\
\text { ) }\end{array}$ & 612 & 10 & $90.30 \%$ & $88 \%$ & $94.44 \%$ \\
\hline $\begin{array}{l}\text { Heart } \\
\text { Disease }\end{array}$ & 270 & 14 & $83.70 \%$ & $78 \%$ & $92.96 \%$ \\
\hline
\end{tabular}

The comparative accuracy of two algorithms are given using Table 3 shows the better performance of VPRSBRRF Classifier than FID3 algorithm. According to the evaluated results the performance of the proposed algorithm is much better as compared to other algorithm.

\subsection{Time Consumption}

The amount of time consumption required to developing data model using proposed algorithm is as on following datasets. Time consumption means time complexity of the algorithm on various datasets.

The comparative time complexity of algorithms are given using Table 4 shows the better performance of VPRSBRRF Classifier than FID3 algorithm

Table 4: Time Consumption of FID3 and VPRS Boundary Region based Random Forest Classifier

\begin{tabular}{|l|l|l|l|l|l|}
\hline Datasets & $\begin{array}{l}\text { Instanc } \\
\text { es }\end{array}$ & $\begin{array}{l}\text { Attr } \\
\text { ibut } \\
\text { es }\end{array}$ & $\begin{array}{l}\text { FID3 } \\
\text { Time } \\
\text { Consum } \\
\text { ption } \\
\text { (In } \\
\text { Seconds } \\
\text { ) }\end{array}$ & $\begin{array}{l}\text { J48 } \\
\text { TC } \\
\text { In } \\
\text { Sec }\end{array}$ & $\begin{array}{l}\text { VPRSBRR } \\
\text { Fime } \\
\text { Consumpti } \\
\text { on } \\
\text { (In } \\
\text { Seconds) }\end{array}$ \\
\hline Iris & 150 & 4 & 0.03 & 0.02 & 0.02 \\
\hline $\begin{array}{l}\text { Wine } \\
\text { Breast } \\
\text { Cancer } \\
\begin{array}{l}\text { Wiscon } \\
\text { sin } \\
\text { (Origin } \\
\text { al) }\end{array}\end{array}$ & 612 & 10 & 0.06 & 0.06 & 0.05 \\
\hline $\begin{array}{l}\text { Heart } \\
\text { Disease }\end{array}$ & 270 & 14 & 0.03 & 0.02 & 0.02 \\
\hline
\end{tabular}

\section{CONCLUSION AND FUTURE WORKS}

The conclusion of entire study about the decision tree algorithms and their methods of performance enhancement. Based on the different experimentations and design aspects some essential points are notices which provided as decision of research work additionally some future extension of the presented work is also provided.

Decision tree algorithm is classical approach of supervised machine learning plus data mining. There are a number of decision tree algorithms are available such as ID3, C4.5 and others. The decision tree algorithms are able to develop a transparent and reliable data model. In order to maintain the transparency and relativity between attributes decision tree algorithms are computationally expensive in terms of memory and time, As a result number of approaches are grown in current years by which the classifiers are claimed to provide much efficient classification accuracy in less complexity. To overcome these computationally expensive in your proposed approach.

In this presented work, feature selection is done by using Variable Precision Rough Set with Boundary Region and decision tree is constructed by Random Forest Classifier. By combining this approach a new VPRSBRRF Classifier is proposed and implemented. The proposed algorithm is enhancing classification accuracy of datasets, reducing the size of tree and minimizing the redundancy in data.

The proposed model is implemented using WEKA 3.7.2 and MATLAB R2015b and the comparative study is performed with respect to the FID3 algorithm and VPRSBRRF Classifier. The comparison among these algorithms is performed in case of accuracy and time complexity. The comparative performance is as following in the table 6.1.

The proposed algorithm, VPRSBRRF produces high accuracy, low error rate and consumes less time as compared with FID3 algorithm. Thus proposed algorithm provides efficient and effective results for classification of datasets.

Table 5: Performance Summary

\begin{tabular}{|c|c|c|c|}
\hline $\begin{array}{c}\text { S. } \\
\text { No. }\end{array}$ & Parameters & $\begin{array}{c}\text { Proposed } \\
\text { VPRSBRR } \\
\text { F Classifier }\end{array}$ & $\begin{array}{c}\text { FID3 } \\
\text { Algorithm }\end{array}$ \\
\hline 1 & Accuracy & High & Low \\
\hline 2 & $\begin{array}{c}\text { Time } \\
\text { Consumed }\end{array}$ & Low & High \\
\hline
\end{tabular}

\subsection{Future Work}

The proposed algorithm is efficient and accurate which provides effective results as compared to the traditional algorithms. In future we will optimize the performance of classification in terms of memory consumption and training time. In future we will parallel this algorithm for analysis of big data.

\section{REFERENCES}

[1] Nguyen H S, Skowron A. Quantization of real value attributes. Proceedins of Second Joint Annual Conf. on Information Science, Wrightsville Beach,North Carolina,pp34-37,1995 
[2] Study on discretization in rough set based on genetic algorithm cai-yun chen, zhi-guo li, sheng-yong qiao, shuo-pin wen Center for Combinatorics, LPMC, Nankai University, Tianjin, 300071, P. R. China E-MAIL: sbickle@eyou.com

[3] Z. Pawlak, Rough sets, Int. J. Comput. Inform. Sci. 11 (1982) 341-356.

[4] Discretization Techniques: A recent survey Sotiris Kotsiantis, Dimitris Kanellopoulos Educational Software Development Laboratory Department of Mathematics, University of Patras, Greece, GESTS International Transactions on Computer Science and Engineering, Vol.32 (1), 2006, pp. 47-58

[5] Rough Sets, their Extensions and ApplicationsQiang Shen_ Richard Jensen. 04(1), January 2007, 100-106 DOI: $10.1007 / \mathrm{s} 10453-004-5872-7$

[6] W. Ziarko. Variable Precision Rough Set Model. Journal of Computer and System Sciences, vol. 46, no. 1, pp. 39$59,1993$.

[7] F. Jiang, Z.X. Zhao,Y. Ge, A supervised and multivariate discretization algorithm for rough sets, in: Proc. of the 5th International Conference on Rough Set andKnowledge Technology, LNCS, vol. 6401, 2010, pp. 596-603.

[8] KDD Cup $99 \quad$ Dataset, 1999. <http://kdd.ics.uci.edu/databases/kddcup99/kddcup99.ht $\mathrm{ml}>$.
[9] R. Kerber, Chimerge: discretization of numeric attributes, in: Proc. of the Ninth National Conf. of Articial Intelligence, 1992, pp. 123-128

[10] Z. Pawlak, Rough Sets: Theoretical Aspects of Reasoning about Data, Kluwer Academic Publishers, Dordrecht, 1991

[11] G.Y. Wang, Rough Set Theory and Knowledge Acquisition, Xian Jiaotong University Press, 2001

[12] https://archive.ics.uci.edu/ml/datasets.html

[13] A Comparative Study of Discretization Methods for Naive-Bayes Classifiers Ying Yang1 \& Geoffrey I. Webb2 1 School of Computing and Mathematics Deakin University, VIC 3125, Australia yyang@deakin.edu.au 2 School of Computer Science and Software Engineering Monash University, VIC 3800, Australia Geoff.Webb@mail.csse.monash.edu.au

[14] "A novel approach for discretization of continuous attributes in rough set theory "Feng Jiang a, $\Uparrow$, Yuefei Su b College of Information Science and Technology, Qingdao University of Science and Technology, Qingdao 266061, PR China b Key Laboratory of Intelligent Information Processing, Institute of Computing Technology, Chinese Academy of Sciences, Beijing 100080, PR China. Knowledge-Based Systems 73 (2015) 324-334.

[15] "Random Forests "Leo Breiman Statistics Department University of California Berkeley, CA 94720 January 2001 